\title{
Volume Rendering Technique (VRT) for Planning and Learning Cranio-Vertebral Junction (CVJ) Surgeries: Technical Note
}

\author{
Kartikeya SHUKLA ${ }^{1}$, Rajnish K PATIDAR ${ }^{1}$, Mayank GARG ${ }^{1}$, Suryanarayanan BHASKAR ${ }^{1}$, Pushpinder KHERA ${ }^{2}$, \\ Pradeep K BHATIA ${ }^{3}$, Deepak K. JHA ${ }^{1}$
}

\begin{abstract}
${ }^{1}$ All India Institute of Medical Sciences, Department of Neurosurgery, Jodhpur, India
${ }^{2}$ All India Institute of Medical Sciences, Department of Diagnostic and Interventional Radiology, Jodhpur, India

${ }^{2}$ All India Institute of Medical Sciences, Department of Anesthesiology and Critical Care, Jodhpur, India
\end{abstract}

Corresponding author: Mayank GARG mayank.garg81@gmail.com

\section{ABSTRACT}

AIM: To present the application of volume rendering technique (VRT) of computed tomography (CT) scan in cranio-vertebral junction (CVJ) surgeries.

MATERIAL and METHODS: The authors used VRT images of CT scan in patients of congenital CVJ anomalies who were subjected to various surgeries through midline posterior approach. The screw entry points and courses of vertebral arteries were identified in relation to various landmarks on the VRT images, preoperatively and intra-operatively.

RESULTS: A total of 15 patients of CVJ anomalies, who underwent surgeries through midline posterior approach, formed the study group. The screw entry points and courses of the vertebral arteries could be effectively identified in the surgical fields.

CONCLUSION: We conclude that VRT images are an inexpensive and user friendly tool to identify the screw insertion points in the surgeries of CVJ anomalies. They also help in identifying the course of the vertebral artery in the surgical field. On the whole, their use makes the surgery in this region, safer.

KEYWORDS: Cranio-vertebral Junction anomalies, Volume Rendering Technique, Vertebral arteries, Basilar Invagination, Atlantoaxial dislocation

ABBREVIATIONS: CVJ: Cranio-vertebral junction, CMJ: Cervico-medullary junction, CT: Computerized tomography, 3D: 3-Dimensional, VRT: Volume rendering technique, MPR: Multiplanar reconstruction, OR: Operating room, VA: Vertebral artery

\section{INTRODUCTION}

$\mathrm{T}$ The cranio-vertebral junction (CVJ) has a complex anatomy and surgery in this location has got its own risks, which include neurovascular injuries, especially of the vertebral artery and the cervico-medullary junction (CMJ) $(7,13,14)$. Such risks are further enhanced when the $\mathrm{CVJ}$ is anomalous and needs reorientation/reduction of CVJ using screws into the lateral masses/ pedicles of the cervical vertebrae, C1 and C2, for fixation to facilitate fusion, especially in case of variations in osseous and vascular anatomies of C1-C2 and the vertebral artery course, respectively (8).
Various tools and techniques have been used to safeguard vertebral artery injuries in the surgeries of CVJ, which include Fluoroscopy, O-arm, intraoperative Computerized Tomography (CT) scan, Neuronavigation and more recently, 3-Dimensional (3D) printing, to better understand the anatomy and use it as a reference during surgery $(3,5,10,11)$. Volume Rendering Technique (VRT) has been used in various surgical disciplines like Facio-maxillary, Cardio-thoracic and orthopaedics disciplines $(2,4,9)$. However, there is no report highlighting its application in CVJ surgeries. The authors share their experience of using VRT in CVJ surgeries done thorough midline posterior approach.
Kartikeya SHUKLA (10): 0000-0002-4051-1652

Rajnish K PATIDAR (1) : 0000-0002-4473-3009

Mayank GARG (1) : 0000-0001-5574-366X
Suryanarayanan BHASKAR (1) : 0000-0001-6001-0217

Pushpinder KHERA

Pradeep K BHATIA

(D) : $00000-0002-9714-5462$
Deepak K. JHA (1) : 0000-0002-4331-9084 


\section{MATERIAL and METHODS}

It was an observational study, done from January 2019 till August 2020, approved by the institute ethics committee. It included all patients undergoing surgery for anomalous CVJ region. CT scans of CVJ with angiography were done on the CT scanner (Somatom Definition Flash, 256 slice, Siemens, Germany). Continuous or overlapping slices, with slice thickness of $1 \mathrm{~mm}$ with zero gap, were used for imaging studies, to obtain the image quality required for adequate $3 D$ visualization and surgical planning. The VRT images were seen on Radiant Dicom Viewer (64 bit, version 4.6.0, Medixant, Promienista 25, 60-288, Poznań, Poland). The images were seen and edited, as mentioned in the article by Jha et al. (6). The surface specific structures like pits, crests, grooves and ridges over the bones (occiput, atlas, axis vertebrae) were identified on the VRT images. The screw entry points for occiput/lateral masses/pedicles were identified based on morphological features mentioned above. The gross direction of the screws and inter-facetal spacers (for C1-2 joint spaces) could be ascertained based on VRT images. However, the detailed dimensions of the screws and inter-facetal spacers were ascertained based on 3D MPR (Multiplanar Reconstruction) CT images in the appropriate cut sections. The length and diameters were measured using the 'measurements and tools' preset.

After adequate surgical exposure of the occiput, C1, C2 or lower level vertebral (as per indication) spines / laminae and lateral masses, predetermined landmarks identified on VRT images were matched with exposed bony structures. Four to five landmarks were identified for intraoperative identification. The rest of the surgery was done as per the merit of the pathology. The screw entry points were selected preoperatively, in relation to the various morphological landmarks. However, the dimensions (diameter and length) of the screws and inter-facetal spacers (length, width and height) were matched with the 3D MPR images. Intraoperative fluoroscopy was used throughout the surgical procedure, as and when needed. Post-operative CT scan of CVJ was done to assess placements and positions of the implants, using both 3D MPR and VRT images, in addition to conventional 2D axial images. The reconstructed 3D VRT images were seen on a laptop computer, by the operating surgical team in Operating Room (OR).

\section{RESULTS}

A total of 15 patients, including 8 males and 7 females were studied. The details of the study population, which include demographic, clinical and intraoperative details as well as the landmarks obtained from VRT, are shown in the table. All the morphological landmarks identified on the VRT images could be verified in the surgical fields. It helped in joint remodeling and the placement of spacers into $\mathrm{C} 1-2$ joint space through the midline posterior approach, where a direct view of anterior structures was limited (Figure 1A-C).

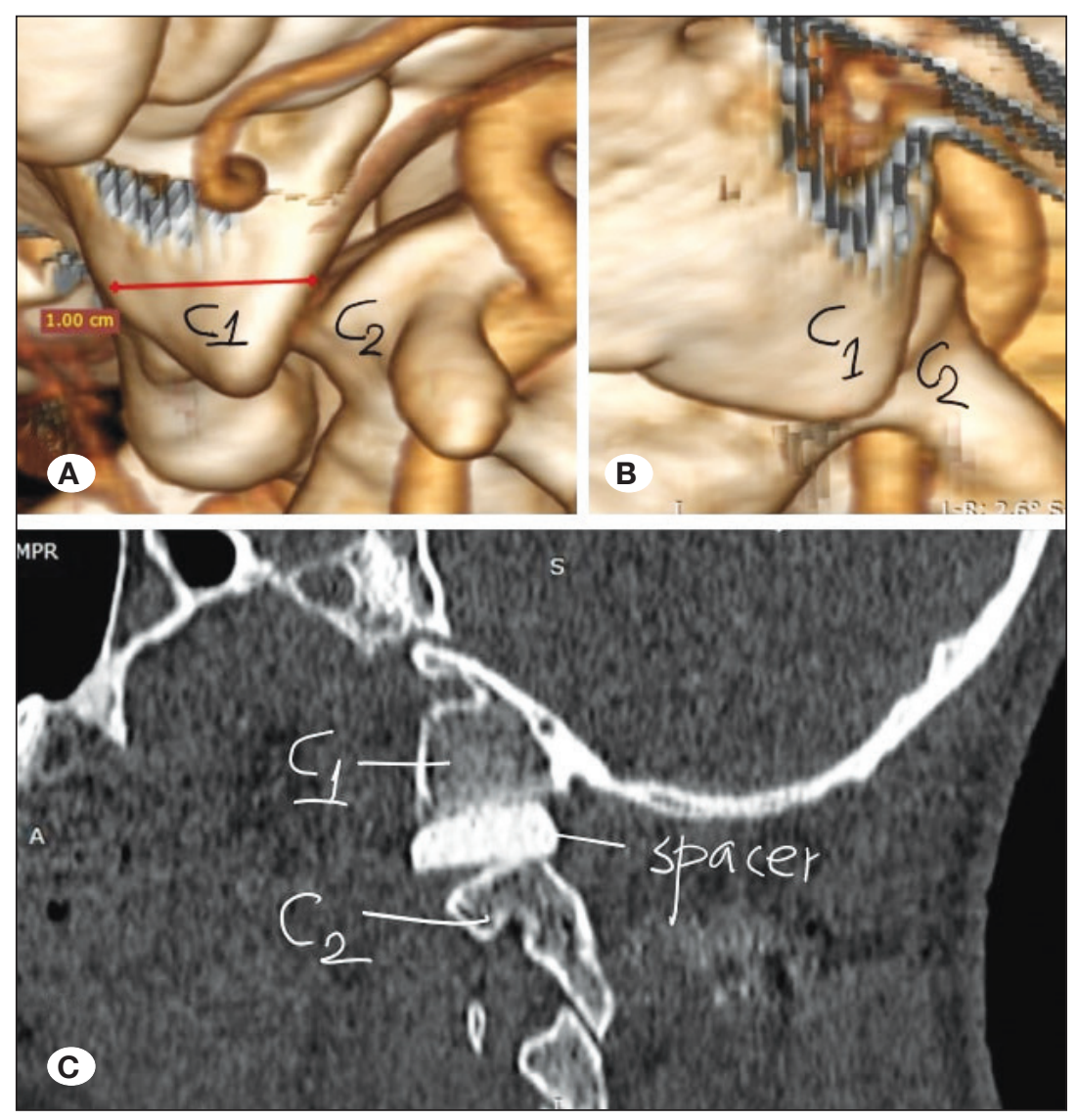

Figure 1: A) VRT sagittal view of dislocated C1-C2 joint, B) VRT anterior view of $\mathrm{C} 1$ and C2, C) Post-operative MPR image (left parasagittal section) of $\mathrm{C} 1$ and $\mathrm{C} 2$ joint with spacer in situ. Joint remodeling done by removing $1 \mathrm{~cm}$ of lower part of $\mathrm{C} 1$. 


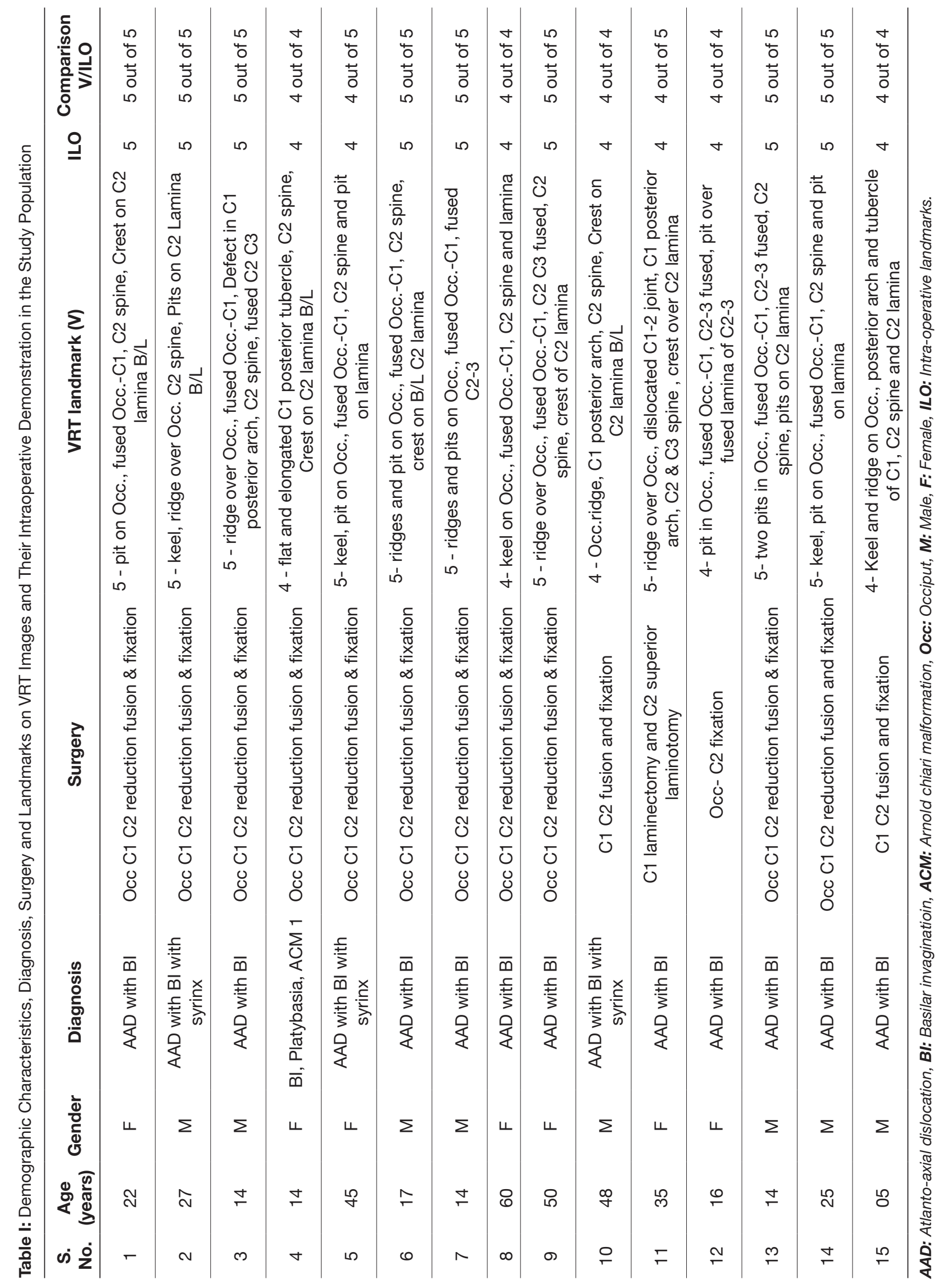


VRT images of the Vertebral Artery (VA) were helpful in each case to avoid its injury during the soft tissue dissection around the $\mathrm{C} 1-\mathrm{C} 2$ facets. Although the vertebral arteries were neither directly exposed nor injured in any of the cases, nevertheless, their courses were confirmed using vascular doppler (Mizuho $20 \mathrm{MHz}$ Surgical Doppler System, Tokyo, 113-0033, Japan) during soft tissue dissection. (Figures $2 A, B$ and $3 A, B$ ) The screw entry points, selected in the VRT images preoperatively, were found in all the cases during surgery based on the identified landmarks. However, the diameters and lengths of the implants (screws and inter-facetal spacers) were decided preoperatively, based on 3D MPR Images of CT scan and were consistently found accurate. The VRT images were also found effective in the post-operative assessment of the remodeled CVJ along with the implants (Figure 4A, B).

\section{DISCUSSION}

The CVJ is considered to have a complex anatomy. Anomalies of this region lead to various types of CVJ instabilities which need surgical realignment of the joint and fixation using screws and rods. Vertebral artery injury is the most feared complication which has deterred many young neurosurgeons, not to operate upon these patients $(3,9)$. The recent use of patient specific 3D printed models of CVJ as a pre-operative and intra-operative reference has provided a means to understand the complex anatomy of CVJ (5). The most commonly used methods of 3D printing for medical models are Fused Deposition Modelling (FDM), Stereolithography (SLA) and Selective Laser Sintering (SLS) (1). All of these techniques take approximately 12-24 hours for printing a real size CVJ model, which includes part of occiput and C1 to C4 or C5. The cost of such a 3D printed CVJ model ranges from 150 to 1000 USD, depending on the technology used (6). The authors used Radiant DICOM Viewer software to see the VRT images on personal desktop/laptop computers. The trial version of this software is available for free and the licensed version costs approximately 15 USD. The ease of feasibility, to remove parts of the CVJ (akin to virtual surgery) of the VRT image, provides the liberty to investigate the regions covered by the overlying structures.

Various methods of intraoperative visualization of CVJ like fluoroscopy, O-arm, Intra-operative CT scan and neuronavigation can be helpful only if, the surgeon is fully aware
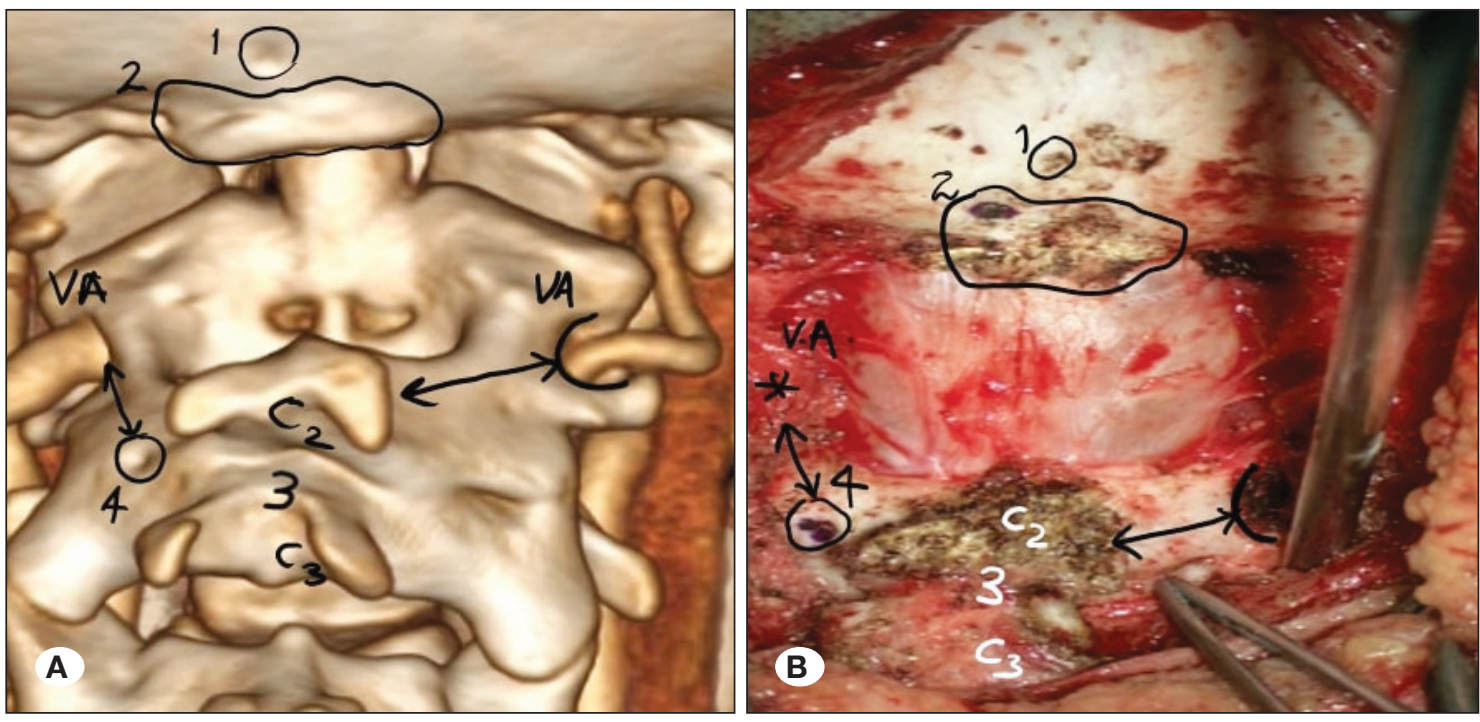

Figure 2: A) VRT reconstructed CT angiography of CVJ showing 4 bony landmarks numbered 1 to 4 , right Foramen Transversarium with right and left vertebral arteries B) Intraoperative Image showing the landmarks as in VRT image.
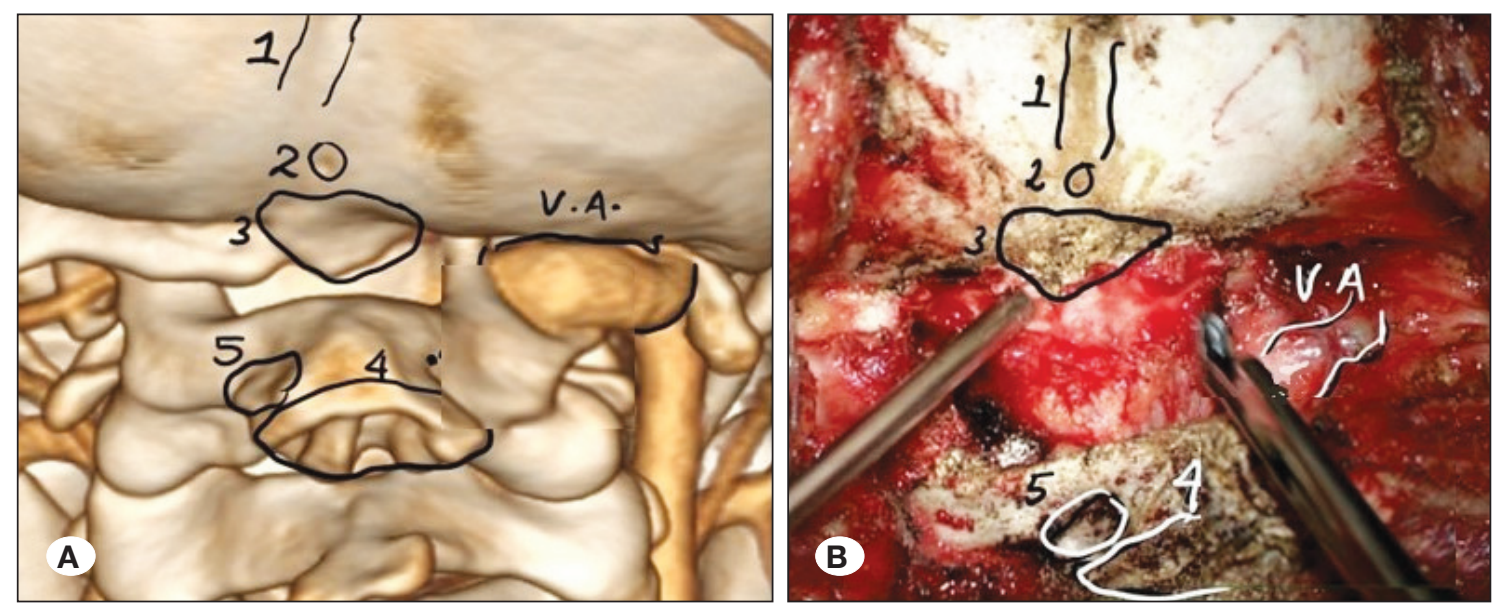

Figure 3: A) VRT images of CT angiography of CVJ showing 5 bony landmarks numbered 1 to 5 . Right vertebral artery shown in relation to the $4^{\text {th }}$ landmark B) Intra-operative Image showing the landmarks as in VRT image. 

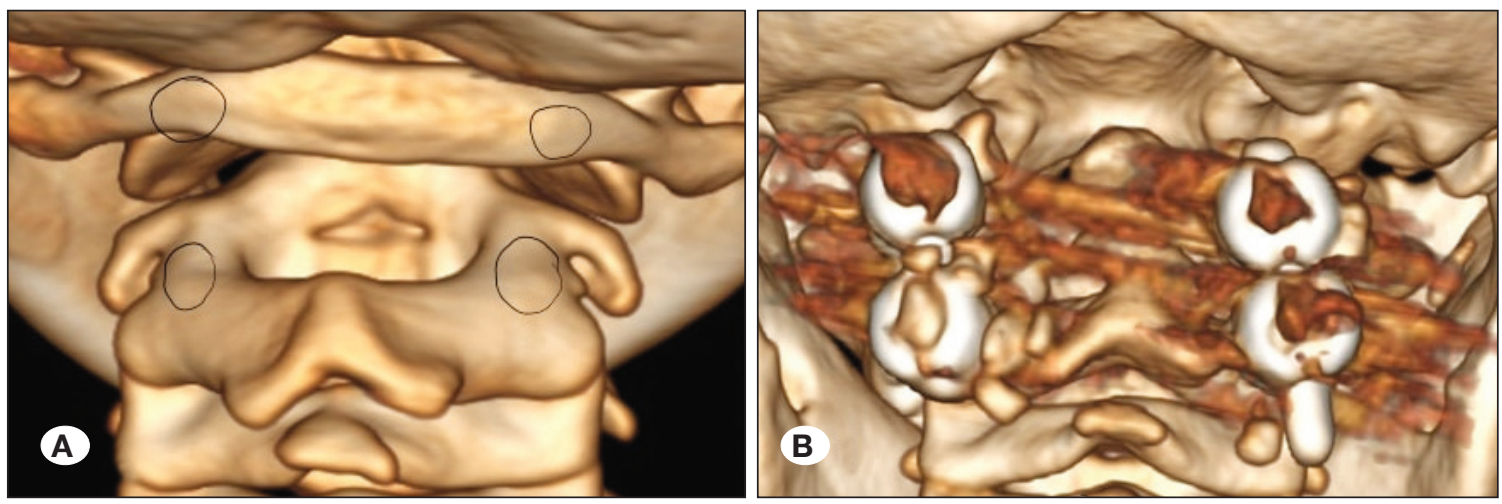

Figure 4: A) Preoperative VRT image for screw entry points of $\mathrm{C} 1$ and C2, B) Postoperative VRT image showing obscured entry points and implant.

of the morphologies of the structures and their orientation in relation to each other. The surgical exposure requires an orientation of, not only the visualized parts of occiput, $\mathrm{C} 1$ and $\mathrm{C} 2$ but also that of a few non-visualized structures like the anterior parts of $\mathrm{C} 1$ arch, the lateral masses of $\mathrm{C} 1-\mathrm{C} 2, \mathrm{C} 2$ pedicle, C2 vertebral body and most of the segments of the vertebral arteries. Surgical manipulations alter the positions of these structures to further complicates the anatomy. Authors do feel that the surgeons who want to operate CVJ, must have clear concepts of the normal anatomy of this region and only then it will be feasible for them to understand the anomalous anatomy or the anatomy distorted by the disease processes. The VRT images give immediate information, which is available on a personal computer/ laptop without any cost implication. The surface morphologies like crevices, depression, foramina and the undulation over the structures exposed during the surgery, can provide important information regarding structures underneath, the screw insertion points, the trajectories and orientations of the screws to be inserted for the reduction of CVJ and subsequent fixations. As in the VRT images of our two study groups, the bony landmarks over Occiput (keel, pits and ridges), deficient posterior arch of C1, the fused occiput and $\mathrm{C} 1$, the spinous processes of $\mathrm{C} 2, \mathrm{C} 3$, the crests over lamina of $\mathrm{C} 2$, were all used to mark the entry point and the trajectory of the implants.

The entry site for the screws is one of the most important step for safe instrumentation of CVJ. The physical dimension (diameter/length) and the direction of the screws are equally important, but the direction can never be objectively confirmed in a CVJ surgery due to its mobility during surgery (12). The entry sites over the Occiput/C1/C2 and the physical dimensions of the screws could be objectively ascertained preoperatively, with the help of the VRT images (screw entry sites) and 3D MPR images (Physical dimensions of the screws and inter-facetal spacers). The authors found that the 3D MPR images in conjunction with the 3D VRT images are quite effective in safe instrumentation of CVJ and also in the postoperative assessment of CVJ.

We conclude that the VRT images are an inexpensive tool which can be used on a personal desktop/laptop computer. The VRT images are effective in objectively identifying screw entry sites in the surgeries of CVJ. Combined with the 3D MPR images, they help in the objective assessment of the physical dimensions of the screws and inter-facetal spacers used for CVJ fixation surgeries.

\section{ACKNOWLEDGEMENT}

Authors acknowledge Mr. Mohan Singh, CT technologist, for technical support in operating the Radiant Dicom Viewer and help in CT angiography examination.

\section{REFERENCES}

1. Aimar A, Palermo A, Innocenti B: The role of $3 D$ printing in medical applications: A state of the art. Journal of Healthcare Engineering 2019:5340616, 2019

2. Calhoun PS, Kuszyk BS, Heath DG, Carley JC, Fishman EK: Three-dimensional volume rendering of spiral CT data: Theory and method. Radiographics 19(3):745-764, 1999

3. Cokkeser Y, Naguib MB, Kizilay A: Management of the vertebral artery at the craniocervical junction. Otolaryng Head Neck 133(1):84-88, 2005

4. Cutroneo G, Bruschetta D, Trimarchi F, Cacciola A, Cinquegrani M, Duca A, Rizzo G, Alati E, Gaeta M, Milardi D: In vivo CT direct volume rendering: $A$ three-dimensional anatomical description of the heart. Pol J Radiol 81:21-28, 2016

5. Du YQ, Qiao GY, Yin YH, Li T, Tong HY, Yu XG: Usefulness of 3D printed models in the management of complex craniovertebral junction anomalies: Choice of treatment strategy, design of screw trajectory, and protection of vertebral artery. World Neurosurg 133:e722-e729, 2020

6. Jha DK, Khera P, Bhaskar S, Garg M: Three-dimensional volume rendering: An underutilized tool in neurosurgery. World Neurosurgery 130:485-492, 2019

7. Lopez AJ, Scheer JK, Leibl KE, Smith ZA, Dlouhy BJ, Dahdaleh NS: Anatomy and biomechanics of the craniovertebral junction. Neurosurgical Focus 38(4):E2, 2015

8. Muthukumar N: Problems in instrumentation of syndromic craniovertebral junction anomalies - case reports. Neurospine 16(2):277-285,2019

9. Pretorius ES, Fishman EK: Volume-rendered three-dimensional spiral CT: Musculoskeletal applications. Radiographics 19(5):1143-1160, 1999

10. Randazzo M, Pisapia JM, Singh N, Thawani JP: 3D printing in neurosurgery: A systemic review. Sur Neurol Int 7 Suppl 33: S801-S809, 2016 
11. Salmi M, Paloheimo KS, Tuomi J, Wolff J, Makitie A: Acuuracy of medical models made by additive manufacturing (rapid manufacturing). J Craniomaxillofac Surg 41(7):603-609, 2013

12. Salunke P, Futane S, Sahoo SK, Ghuman MS, Khandelwal N: Operative nuances to safeguard anomalous vertebral artery without compromising the surgery for congenital atlantoaxial dislocation: Untying a tough knot between vessel and bone. J Neurosurg Spine 20(1):5-10, 2014
13. Song GC, Cho KS, Yoo DS, Huh PW, Lee SB: Surgical treatment of craniovertebral junction instability: Clinical outcomes and effectiveness in personal experience. J Korean Neurosurg Soc 48(1):37-45, 2010

14. Yamazaki M, Okawa A, Furuya T, Sakuma T, Takahashi H, Kato K, Fujiyoshi T, Mannoji C, Takahashi K, Koda M: Anomalous vertebral arteries in the extra-and intraosseous regions of the craniovertebral junction visualized by 3-dimensional computed tomographic angiography: Analysis of 100 consecutive surgical cases and review of the literature. Spine (Phila Pa 1976) 37(22):E1389-1397, 2012 